\title{
To What Extent do Fracture Clinic Patients use Smart Mobile Technology and What are Their Specific Educational and Rehabilitee Needs that can be Addressed
}

\author{
Balasubramanian Balakumar ${ }^{1}$, Neil Einstein ${ }^{2}$, Paul Hindle ${ }^{3}$ and Rajpal Nandra ${ }^{1 *}$ \\ ${ }^{1}$ Specialty Registrar, Queen Elizabeth Hospital, Birmingham, UK \\ ${ }^{2}$ Specialty Registrar T\&O, PhD Researcher, University of Birmingham, UK \\ ${ }^{3}$ Wing Commander, Consultant Orthopaedic Surgeon, Queen Elizabeth Hospital, UK \\ ${ }^{*}$ Corresponding author: Rajpal Nandra, Specialty Registrar, Queen Elizabeth Hospital, Birmingham, UK
}

Received: June 17, 2020; Accepted: July 15, 2020; Published: July 27, 2020

\begin{abstract}
Introduction: The fracture burden of the UK utilises a vast proportion of National Health Service (NHS) resources. Subsequent complications in healing result in poor patient outcomes (indirect costs) and increased demand on healthcare services (direct costs). Inadequate education regarding risk factors for poor outcomes provides a target for intervention. The increasing proportion of smartphone users makes smartphone applications (apps) a viable platform from which to distribute educational resources and conduct research.
\end{abstract}

Methods: A questionnaire was distributed randomly to 100 patients attending fracture clinic at the Queen Elizabeth Hospital in Birmingham over a twelve-week period. The mean age was 46 years (range 19 to 78), 52\% female and a third were aged over 60 years. Primary questions determined the proportion of smartphone users, specifically those willing to utilise apps as an educational resource. Secondary information collected included patients' concerns, smoking status, interest in smoking cessation and awareness of the risk factors affecting fracture healing.

Results: Almost $72 \%$ of responders used a smartphone, $71 \%$ would use an app for education and $74 \%$ would allow their data to be utilised for research. Some $60 \%$ of smokers would engage with cessation therapy through an app. The two greatest concerns identified were healing time (46\%) and the longterm consequences of a fracture (46\%). NSAID use was reported in $30 \%$, however only $20 \%$ identified these as risk factor.

Conclusion: The majority of fracture clinic patients use smartphones and are were willing to utilise apps for both healthcare education and research. This could provide a cost-effective solution to an existing void in patient awareness. Developing an out-patient data collection tool offers new opportunities to epidemiological researchers.

Keywords: Fracture, Smartphone, Non-union, Education; Virtual clinic

\section{Introduction}

The incidence rate of fractures in the United Kingdom is approximated at 3.6 per 100 people per year [1]. According to recent statistics from Public Health England, the number of people aged 65 and over will increase by $40 \%$ over the next 17 years. In any year, $35 \%$ of these individuals will have a fall, and $5 \%$ of these falls will result in a fracture [2]. Fractures however, are not unique to the elderly; evidence suggests that almost one third of children will experience at least one fracture before the age of 17 [3]. This rising burden presents an immense challenge to existing NHS services striving to deliver urgent, definitive and complete follow-up care [4].

The impact on healthcare services is further increased by complications of fracture treatment, which require extended periods of follow-up and in some cases, readmission (direct costs). These complications affect up to $10 \%$ of patients within the two years of the initial injury [5]. Patients affected by complications have reported a reduction in their quality of life and a failure to return to their premorbid activity levels (indirect costs) [5,6]. Increased risk of delayed union and non-union has been associated with multiple risk factors. Whilst some, such as age, ethnicity and gender are non-modifiable, others, including prolonged NSAID use, smoking and poorly regulated diabetes management are potentially modifiable. These modifiable factors provide therapeutic targets for intervention [7-9].

Increasingly busy fracture clinics impede clinician's ability to deliver information on fracture rehabilitation and meaningful dialogue on modifiable risk factors. This setting hinders the clinician's ability to address patient concerns, such as answering individualised questions on healing times and functional impairment. As fracture clinics evolve, we see reduced face to face encounters with patients, the introduction of physician assistants and virtual clinics. Improved selfmanagement following a fracture has the potential to improve patient 
outcomes and reduce the strain on healthcare services. Whilst there is extensive evidence for the relationship between modifiable risk factors and fracture healing, there is limited evidence surrounding public awareness of these. Without first establishing and improving public awareness, it follows that limited progress will be made in reducing the number of poor outcomes following acute fractures.

Over recent years, the number of individuals with access to a smartphones has risen exponentially [10]. A recent systematic review investigated the use of healthcare related smartphone applications by both medical professionals and patients. Findings suggested that these applications had a vital role to play in patient education, disease management and the remote monitoring of patients [11]. Considering the incidence of fractures within the UK population and the ability to therapeutically target modifiable risk factors, it is therefore surprising that to date, no smartphone application exists to educate the public on these risk factors and to give advice about fracture management. As a result, we hypothesise that current patient education regarding risk factors may be improved via education delivered through a smartphone application.

This study therefore aims to quantify the possession of smartphones in patients attending fracture clinic, and secondarily, report awareness of risk factors for fracture healing and attitudes towards the use of smartphone applications in acute fracture management.

\section{Method}

Over a twelve-week period, from June to August 2017, 144 patients attending fracture clinic at the Queen Elizabeth Hospital in Birmingham were asked to complete a questionnaire regarding their acute fracture in the waiting room. Of those approached, 100 voluntarily returned the completed forms. All patients seen in the fracture clinic for a first or follow-up appointment following both surgical and conservative management were included. The host hospital is a major trauma centre treating skeletally mature patients and comprises a limb reconstruction service. Patients not admitted via the emergency department are seen within 24 to 48 hours of their injury in fracture clinic. Skeletally immature patients were excluded as they are assessed at the regional Children's' Hospital.

The questionnaire was designed with an ethos of simplicity to encourage compliance and precise questions to reduce data collection bias [12]. Prior to distribution, the questionnaire was peer reviewed by senior orthopaedic clinicians and amendments made. A pilot study was also conducted on a smaller number of patients, to assess feasibility of circulation and the willingness of patients to participate. An adverse event pathway developed in case of any incidents. The final questionnaire comprised of closed and open questions to provide quantitative and qualitative information. The questionnaire included questions relating to patients' concerns about their fracture, smoking status, interest in smoking cessation and understating of the risk factors affecting bone healing. It also recorded their level of smartphone use and willingness to engage with smartphone application in relation to their healthcare (Appendix I).

Data was collated using Microsoft Excel (Office 2016) and statistical analysis was performed using IBM SPSS Statistics 23 . The trusts ethical review board deemed the study to be a service evaluation. No funding was received for this study.

\section{Results}

A total of 100 fracture clinic patients competed the questionnaire over a six-week period. The mean age of participants was 46 years (SD 16.7, range 19 to 78 years). Of the patients who completed the questionnaire $29 \%$ were over 60 years of age and of the responders $52 \%$ were female.

\section{Smartphone Use}

Of the patients who completed the questionnaire, $72 \%$ used a smartphone, of which $52 \%$ owned Apple iPhones $₫$ with IOS Software. Non-smartphone users were predominantly female $(n=26,92 \%)$ and older (mean age 62, range 45 to 76 ). With regards to the role of smartphone application in patient education, $71 \%$ of participants would use an application to educate themselves and support their healing journey, 15 would not and 14 patients did not respond to the question. Similarly, $74 \%$ of participants would be happy for their information to contribute towards research.

\section{Patient Concerns}

The two greatest concerns affecting patients were the time taken for the fracture to heal (46\%) and the long-term consequences of an acute fracture (46\%). Other concerns included functional aspects of the injury such as returning to work, driving and sport. Several patients also commented in free text box worries about the possibility of a recurrent fracture. Figure 1 below details patient concerns following an acute fracture.

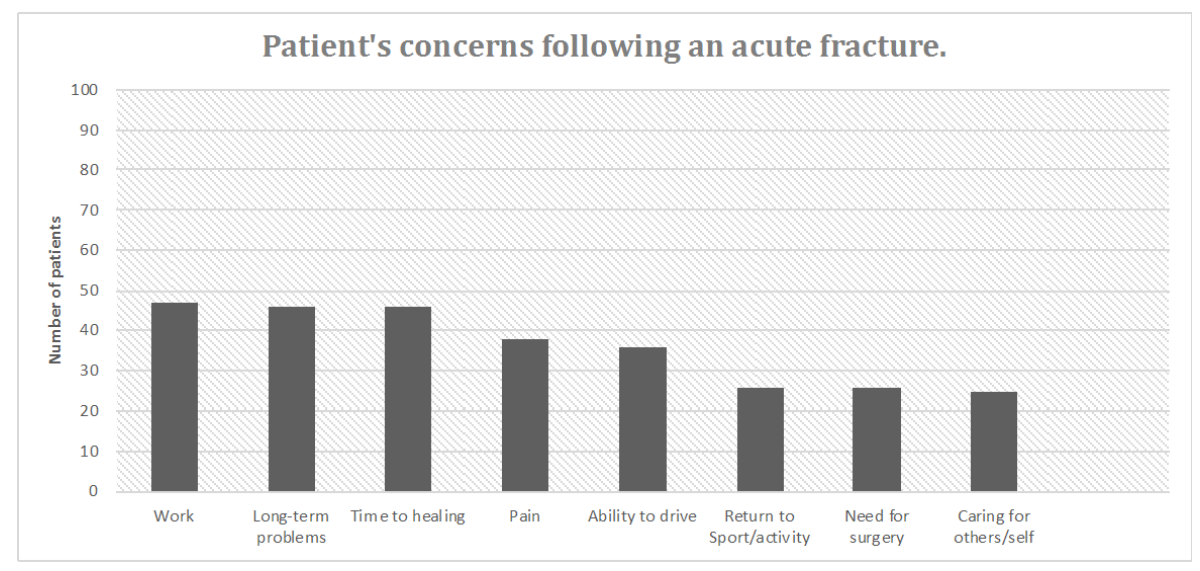

Figure 1: Patient concerns following an acute fracture. 


\section{Smoking}

Of the 25 (25\%) patients who admitted to smoking at the time of being interviewed $60 \%$ stated that they would engage with smoking cessation therapy via a smartphone application. Five respondents would not use smartphone applications to stop smoking and the remainder did not answer $(\mathrm{n}=5)$.

\section{Analgesia}

Of the patients who completed the questionnaire, $30 \%$ admitted to regular use of NSAIDs such as ibuprofen for analgesia. The most commonly used analgesic was paracetamol. Figure 2 shows the analgesia use following acute fracture.

\section{Perceived Risk Factors to Fracture Healing}

There was a wide variety of responses regarding the risk factors associated with poor outcomes following a fracture. There was an increased tendency for the patients to highlight lifestyle factors such as alcohol consumption, smoking, poor diet and obesity, all of which are modifiable. The most commonly recognised risk factor was age (74\%). Other factors such as ibuprofen use (20\%) and diabetes (39\%), were less frequently identified, despite their recognised detrimental effects on fracture healing amongst medical professionals. Interestingly, a select number of participants attributed the use of paracetamol, vegetarian diet and hypertension as risk factors, despite the lack of evidence to substantiate these. Figure 3 below represents the commonly identified risk factors to fracture healing.

\section{Discussion}

This study highlights the wide demographics of patients attending fracture clinic who could benefit from personalised management via smartphone applications, with over three quarters of those questioned having access to such devices. This provides a valuable platform from which to target a large population and opens the door to health interventions delivered via mobile application and web-based tools.

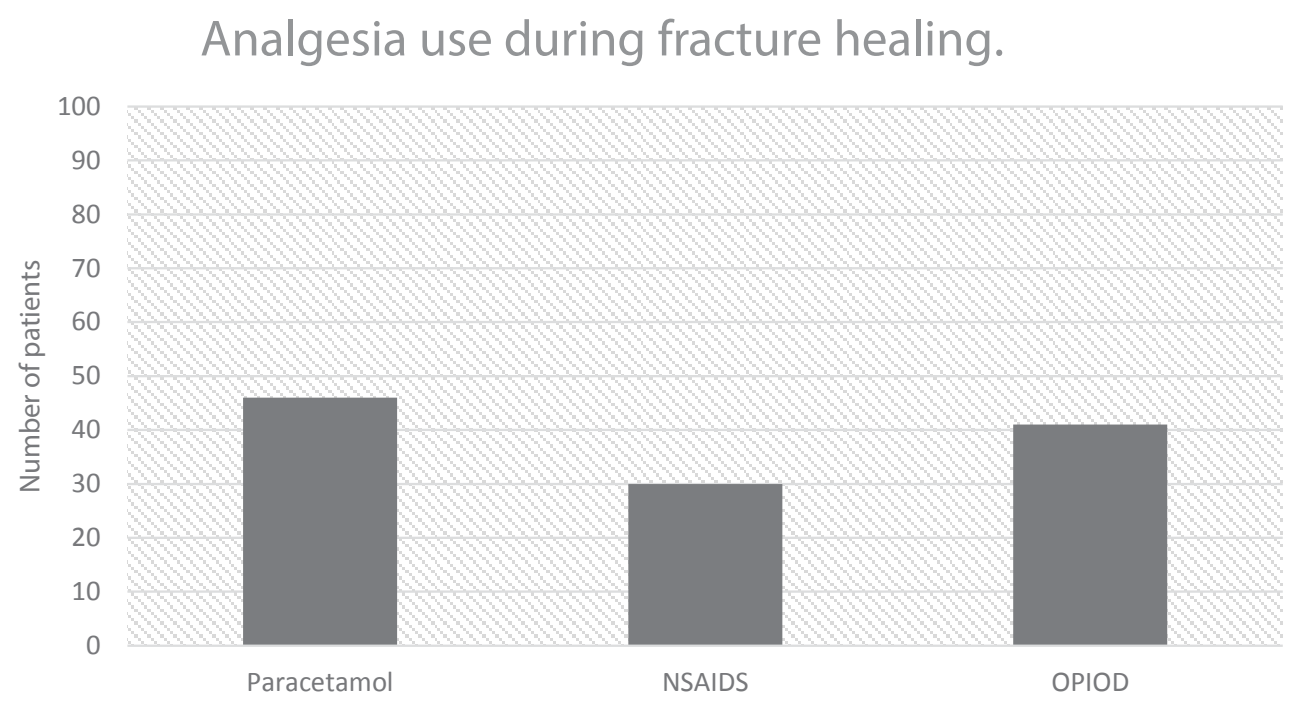

Figure 2: Patient analgesia use following acute fracture.

\section{Factors that affect fracture healing.}

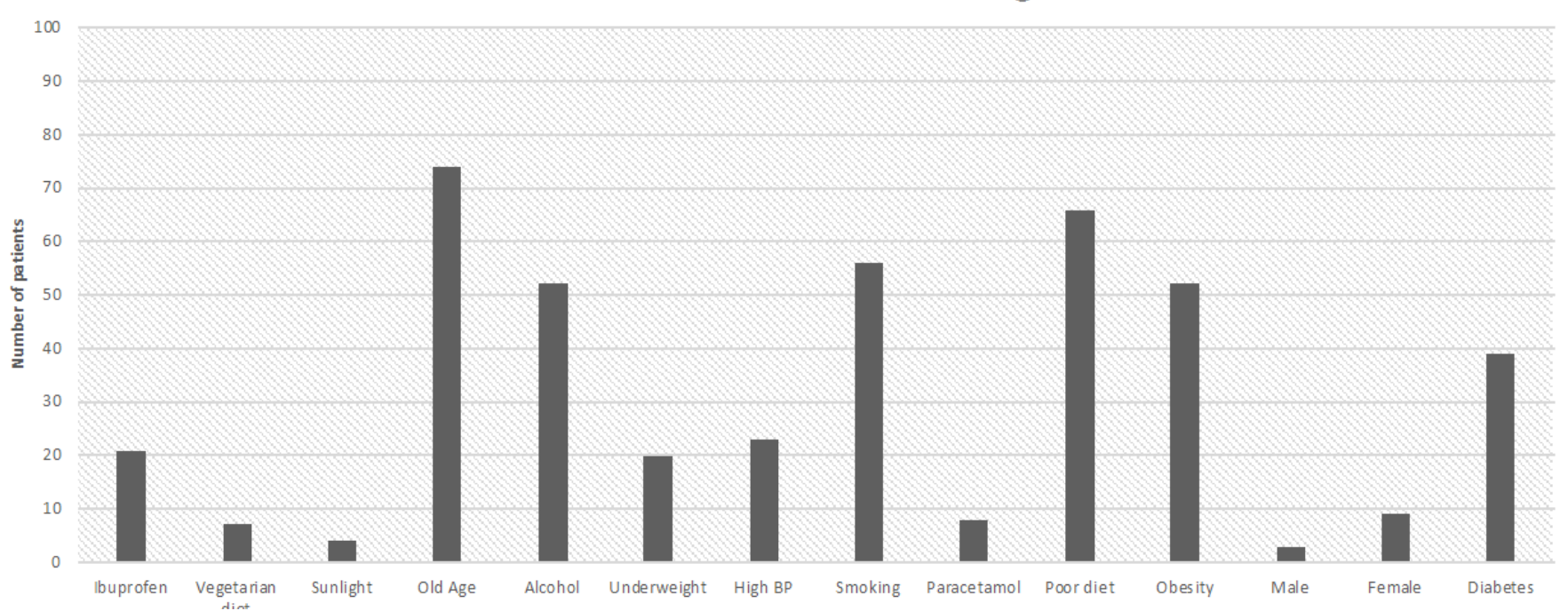

Figure 3: Perceived risk factors to fracture healing. 
Smartphone use was lower in older patients, particularly females, but we anticipate this to increase over the next decade. Correspondingly, $72 \%$ of participants were happy for their anonymised data to be used in research, which could facilitate the development of large, multicentre databases via mobile applications to be used in epidemiological research. Investment by technology industries to create clinician and patient faced applications has increased. There are already devices to help patients regulate blood glucose levels, aid smoking caseation (QuitMedGuide, University of Texas, America) and help treat dementia. Primary care is being delivered remotely via face-to-face mobile platforms (GPatHand, Babylon, London) and clinicians are increasing using smartphones to transfer images, communicate and monitor patient care.

Despite follow-up in fracture clinic, where patient education should be incorporated into the consultation, it is concerning that patient's still lack knowledge of less publicised risk factors such as the prolonged use of NSAIDs and optimising management of chronic diseases. The disparity of the results reinforces our hypothesis that patients lack understanding and need further education to encourage favourable behaviour and promote healing. We have not investigated the causality of poor information delivery but suggest it is sequelae of limited contact time with patients in fracture clinic as clinic sizes increase and resources decrease.

The proportion of smokers within the sample size is representative of the national average of $19 \%$. The latter percentage decreased dramatically from an all-time high of $46 \%$ in 1974 [13]. Despite this relative decrease, smoking remains a considerable factor in non-union $[9,14,15]$. As of 2012, Abroms et al. identified 252 smoking cessation smartphone applications for iPhone and android devices [16]. They suggested that adherence with the applications was poor overall, despite a high download rate. Similar experiences have been reported when modifying other additive behaviours, such as alcoholism, where significant decreases in risky drinking behaviour in alcoholic patients were observed following the use of an application compared to controls [17]. We hypothesise that an acute fracture may provide an opportune moment for successful lifestyle modifications.

Interestingly, patient recognition of risk factors affecting fracture healing demonstrated a trend to identify modifiable lifestyle risk factors such as smoking, obesity and alcohol consumption. These risk factors are in general well publicised by prominent public health campaigns. Established In 2012, the 'Stoptober' campaign has been very successful in raising awareness of the association between smoking and lung cancer [18]. Smoking was identified as a risk factor for poor fracture healing by $53 \%$ of the population questioned, however evidence suggests that smoking is more commonly identified by the population as a risk factor for cancer [19]. These findings highlight the power of patient education and the ability to target vast numbers of people with the aim of improving their self-care. On the other hand, the discrepancy between the public awareness of the effects of smoking on cancer compared to fracture healing suggests that further information must be provided to fracture patients in order for them to make adequate lifestyle modifications.

Introduced in 2009, the NHS campaign 'Change4Life' was targeted specifically at dietary modification to reduce childhood obesity and subsequently promote a healthier nation. A year after its launch, over 400,000 families were participating with an awareness rate of $87 \%$ amongst parents with children in the target age range [20]. Since its development, the campaign has both expanded to incorporate adult health and modernised to utilise a smartphone application providing information on food groups, exercise and recipes. The success of these public health campaigns highlights the scalability of mobile devices and reinforces the importance of patient education as the key to reducing the burden of modifiable risk factors and their consequences on NHS services.

In addition to patient identified risk factors, primary patient concerns included the time taken for the fracture to heal, as well as long term consequences of a fracture. As these figures will vary according between individuals, providing tailored information to patients about appointments, long term follow up and recovery milestones would be of benefit. Utilising the smartphone platform to engage patients would enable them to record their past and upcoming appointments whilst gaining access to information regarding cast care, return to driving and physiotherapy exercises designed to promote rapid return of function. Access to all the necessary resources in one application would act as both a source of information and a method of alleviating patient concerns.

\section{Limitations}

Although a small study, the data collected from this population is consistent with national statistics. However, it is more challenging to compare subjective measures such as level of education and understanding. The sample tested did not include children with skeletally immature fractures. The rate of non-union in children is low and many do not have behaviours that require modification [21]. Considering the lower reading age of children and diminished understanding, they were excluded from the study and the application was tailored towards adults.

Those who failed to return a completed questionnaire were not included in the study, this may constitute an element of selection bias. Conversely, it may be that patients who were more technologically aware were more inclined to complete questionnaire. It would not be able to eliminate this source of bias this unless completion was mandatory. Factors such as reporting and recall bias are also valid considerations. Although the proportion of smokers identified was in keeping with the expected level, some patients may not have reported their smoking habits truthfully.

\section{Conclusion}

Overall, this preliminary research has demonstrated the widespread use of smartphones within the patient population attending fracture and the willingness of patients to engage with healthcare information using this platform. It has also confirmed that the majority of patients would accept having their anonymised data utilised for research purposes, which could facilitate the development of large, multi-centre databases via mobile applications.

A large proportion of patients were unaware of behaviours that impaired fracture healing and focused their concerns on finite endpoints, 
time to healing, ability to drive and long-term morbidity. Reinforcing the need for a more targeted approach to patient management following a fracture, to address these specific agendas. This study provides evidence to support the development of software that will improve overall treatment satisfaction, educate patients and modify behaviour that may in term improve fracture care outcomes. For example, mobile devices can play a vital role in appointment planning, information on cast care, basic physiotherapy exercises and fracture healing.

\section{References}

1. Donaldson LJ, Reckless IP, Scholes S, Mindell JS, Shelton NJ (2208) The epidemiology of fractures in England. Journal of Epidemiology and Community Health 62: 174. [crossref]

2. England PH. Falls and fracture consensus satement: Supporting commissioning for prevention. 2017.

3. Cooper C, Dennison EM, Leufkens HG, Bishop N, van Staa TP (2004) Epidemiology of childhood fractures in Britain: a study using the general practice research database. Journal of Bone and Mineral Research 19: 1976-1981. [crossref]

4. (NHFD) NHFD (2016) National Hip Fracture Database (NHFD).

5. Ekegren CL, Gabbe BJ, Edwards ER, Steiger Rd, Page R (2016) 791 Incidence, costs and outcomes of non-union, delayed union and mal-union following long bone fracture. Injury Prevention

6. Court-Brown CM, McQueen MM (2008) Nonunions of the proximal humerus: their prevalence and functional outcome. The Journal of Trauma 64: 1517-1521. [crossref]

7. Hernandez RK, Do TP, Critchlow CW, Dent RE, Jick SS (2012) Patient-related risk factors for fracture-healing complications in the United Kingdom general practice research database. Acta Orthopaedica 83: 653-660. [crossref]

8. Jiao H, Xiao E, Graves DT (2015) Diabetes and its effect on bone and fracture healing. Current Osteoporosis Reports 13: 327-335. [crossref]
9. Patel RA, Wilson RF, Patel PA, Palmer RM (2013) The effect of smoking on bone healing: A systematic review. Bone \& Joint Research 2: 102-111. [crossref]

10. Ofcom. The UK is now a smartphone society The communications market report 2015: Ofcom; 2015

11. Mosa ASM, Yoo I, Sheets L (2012) A Systematic review of healthcare applications for smartphones. BMC Medical Informatics and Decision Making 12: 67. [crossref]

12. Edwards P (2010) Questionnaires in clinical trials: Guidelines for optimal design and administration. Trials 11:2. [crossref]

13. (HSCIC) HaSCIC (2016) Statistics on Smoking, Englang.

14. Sloan A, Hussain I, Maqsood M, Eremin O, El-Sheemy M (2010) The effects of smoking on fracture healing. The surgeon: Journal of the Royal Colleges of Surgeons of Edinburgh and Ireland 8: 111-116. [crossref]

15. Castillo RC, Bosse MJ, MacKenzie EJ, Patterson BM (2005) Impact of smoking on fracture healing and risk of complications in limb-threatening open tibia fractures. Journal of Orthopaedic Trauma 19: 151-157. [crossref]

16. Abroms LC, Westmaas JL, Bontemps-Jones J, Ramani R, Mellerson J (2013) A content analysis of popular smartphone apps for smoking cessation. American Journal of Preventive Medicine 45. [crossref]

17. Gustafson DH, McTavish FM, Chih MY, Atwood AK, Johnson RA, et al. (2014) A smartphone application to support recovery from alcoholism: A randomized clinical trial. JAMA Psychiatry 71: 566-572. [crossref]

18. Stoptober Public Health England 2012.

19. Sanderson SC, Waller J, Jarvis MJ, Humphries SE, Wardle J (2009) Awareness of lifestyle risk factors for cancer and heart disease among adults in the UK. Patient Education and Counselling 74: 221-227. [crossref]

20. (BHFNC) TBHFNCfPAaH. Change4Life one year on. 2010.

21. Mills LA, Simpson AH (2013) The risk of non-union per fracture in children. Journal of Children's Orthopaedics 7: 317-322. [crossref]

\section{Citation:}

Balasubramanian Balakumar, Neil Einstein, Paul Hindle and Rajpal Nandra (2020) To What Extent do Fracture Clinic Patients use Smart Mobile Technology and what are their Specific Educational and Rehabilitee Needs that can be Addressed. Integr J Orthop Traumatol Volume 3(2): 1-6. 
Rajpal Nandra (2020) To What Extent do Fracture Clinic Patients use Smart Mobile Technology and What are Their Specific Educational and Rehabilitee Needs that can be Addressed

\section{$\underline{\text { Appendix I - Unify Questionnaire }}$}

The University of Birmingham and Queen Elizabeth Hospital are designing a smartphone app for people with fractures. This app will be called MyFracture and will be tailored to each specific patient and their injury. Please could you help us by answering a few questions? Thank you.

About you:

How old are you?

Are you: Male

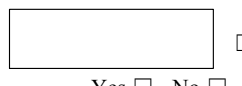

Female $\square$

Do you smoke at all?

Yes $\square$ No $\square$

Do you use a smartphone? Yes $\square$ No $\square$

What type is it? $\quad$ iPhone $\square \quad$ other $\square$

What painkillers have you taken for your fracture?

Questions:

1. What are your main concerns about your fracture? (please tick any that apply)

Getting back to work $\square \quad$ Looking after myself or someone else $\square \quad$ Pain $\square$

Driving $\square \quad$ Getting back to sports / activity $\square \quad$ Long-term problems $\quad \square$

Will I need surgery? $\quad \square \quad$ How long will this take to heal? $\square$

Other:

2. If you are a smoker, would you like the app to help you to quit smoking? (please tick one box)

Yes $\square \quad$ No $\square \quad$ I don't smoke $\square$

Please turn over. More questions on the back $\rightarrow$

3. Please circle the following things that you think can slow down the healing process for broken bones? (please tick any that apply)

Ibuprofen $\square \quad$ Vegetarian diet $\square \quad$ Sunlight $\square \quad$ Old Age $\square \quad$ Alcohol $\square$

Being underweight $\square \quad$ High blood pressure $\square \quad$ Smoking $\square \quad$ Paracetamol $\square$

Poor Diet $\square \quad$ Obesity $\square \quad$ Being male $\square \quad$ Being female $\square \quad$ Diabetes $\square$

Other:

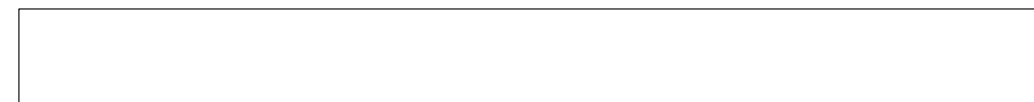

4. What features would you find useful in an app for patients with fractures?

5. If your doctor advised that you needed an operation for your fracture, would you like the app to give you more information about the surgery? (please tick the yes or no box)

Yes $\square$ No $\square$

6. Would you be happy for the app to send our researchers anonymous information about your fracture, your treatment, and your recovery? (please tick the yes or no box)

Yes $\square$ No $\square$

7. Any other comments or ideas?

Thank You - Please put your completed questionnaire in the tray. 